\title{
Practitioner insights on business models and future directions
}

The enviable success of icons such as Facebook, Google and Apple provides irrefutable evidence to managers and entrepreneurs that building innovative and sustainable business models is of paramount importance to surviving and growing their business. Yet, following one of the most severe downturns in the history of our global economy, most are concerned that their business model(s) fails to create and capture sufficient value to meet the radically unpredictable competitive landscape ahead. The result is a clamour of demand for business tools, techniques and specific guidance to help managers and entrepreneurs build and adapt their business models for capturing and creating value.

For several years, we have been engaged with academic networks addressing these issues theoretically, but we noted with concern that few papers translate their findings to address the demands for management guidance, and messages about business models are often confusing and contradictory. Compelled to respond, the eight papers selected for this special issue of the Journal of Business Strategy directly address three challenges critical to managers and entrepreneurs, from start-ups to small- and medium-sized enterprises to multinationals, with a large portfolio of business models. We identified these challenges as: first, the need for managers to be able to evaluate current business models, whether these are based on traditional approaches, freemium or crowdsourcing; second, the need for frameworks to guide the transformation of existing business models to meet identifiable shifts in the business landscape such as digitization; and third, approaches for innovating and creating business models which are new to the firm.

To address this first challenge, a method for systematically assessing the performance of a firm's existing business model(s) is provided by Haggege et al. Their framework explains how managers and entrepreneurs can manipulate five key drivers to enhance business model performance, and presents a suite of questions enabling the evaluation of different configurational combinations to guide managers in achieving better performing business models. While Haggege et al. address models generally, Holm and Gunzel-Jensen reveal measures specifically identified for succeeding with popular freemium business models including Spotify, Linkedln and Box.com that provide a basic version of a product or service for free, with the intention of enticing sufficient numbers of customers to pay for a more advanced version. Holm and Gunzel-Jensen demonstrate how success can be achieved through aligning competitive strategy, business model configuration and strategic choices, focusing on the intricacies of managing the tensions between free and premium options. Strategies for sustaining existing business models are provided by Kohler and Nickel in relation to crowd sourcing platforms. While lauded by the popular press, reliance of the creativity of the crowd for companies like Threadless, which creates value by outsourcing t-shirt design to an art community of 120,000 designers worldwide, no longer demands just a degree of engagement, but strategies to win and nurture the long-term commitment of the crowd.

We then selected three papers to help managers respond to our second identified challenge - forthcoming shifts in the business landscape brought about by the increasing intertwining of business models and digital technology. Benyayer and Kupp provide a practical explanation for and means of evaluating the five major dimensions of opening up a business model (motivation, object, community, actions and governance), and identify the potential strategic responses available to traditional organizations to navigate these new forms of competition. The coming years will see the digitization of many traditional industries such as automotives, logistics, health and energy, and many managers are concerned about the impact of this transformation on their organization's ability to create 
and capture value. In response, Remane et al. provide managers from traditional industries with a blueprint to systematically analyse and discover digital business models. Further addressing the complexities of digital business models for managers, Parmentier and Gandia suggest a set of guidelines for moving an organization from a single-sided business model to a multisided business model such as Airbnb, which connects travellers, renters and service providers through its multisided platform architecture. Their practical framework details the chronological steps available to small- and medium-sized enterprises for redesigning existing single-sided business models to expand and consolidate into multisided models with a platform for products and/or services.

To address the third challenge, we selected two papers with alternative approaches for innovating and creating business models new to a firm. While many business model design tools which exist, such as Business Model Canvas (Osterwalder and Pigneur, 2013) or the Business Model Kit (Board of Innovation, n.d), provide useful templates, these fail to provoke managers to reframe their beliefs, challenge industry norms and generate creative business model ideas. Winterhalter et al. provide first-hand practical revelations on how managers can systematically establish, embed, manage and apply a corporate business model innovation process into existing innovation activities to create and maintain an entire portfolio of different business models for different markets. Rumble and Minto's paper promotes the value of analogies or applying insights from one industry setting to another to stimulate and provoke critical thinking. Their paper reveals how managers can learn about businesses in other arenas and then transfer and interpret these ideas to generate multiple insights for designing business models that are unique in their industry context.

In sum, these eight papers address the key challenges for managers operating in constantly shifting business landscapes. Much as we are convinced that these papers provide valuable insights, our experiences, and the corporate experiences incorporated throughout the related studies, force us to recognize that chasms remain in providing pragmatic guidance for managers wishing to develop business models which are both novel and appropriate for their firms. While we offer no simple solution, we suggest two clear directions for addressing this problem. The first is to note that traditional business model tools and techniques in the form of simple lists and questions resolutely fail to challenge the rules of industry games or shift long-held assumptions to generate innovative business models. Instead, if they are to generate alternative models which anticipate and respond to change, managers must be willing to shift allegiance from these previously successful templates and conventional approaches. Shifting industry mindsets and embedded mental frames to anticipate or even trigger new and emerging realities is painful, particularly when research provides few guidelines as to how managers can shift their mental frames, be free of assumptions and reimagine the foundations of their business.

We then go on to question the value of searching for business model innovation in the sense of innovating business models which are new to the world. We suggest that novelty should be considered not in a world or industry context, but should be specific to the firm or its location. The ability to explore a catalogue of business models adopted in remote and distant industries would, we argue, stimulate reframing and allow managers to utilize existing business models in new contexts. Now that there is relative consensus on the components of business models, data bases or catalogues of case studies capturing the wide range of business models utilized can be developed. Several business schools are already advancing such projects but more needs to be done to catalogue the various configurations of business models and to make these catalogues available to practitioners. Managers and entrepreneurs could then use these catalogues to virtually explore and manipulate alternative business model configurations from sectors and industries around the globe. In essence, these catalogues would expose managers to different business models, encouraging them to not only "borrow" business model configurations which are novel and appropriate to their

PAGE 4 | JOURNAL OF BUSINESS STRATEGY | VOL. 38 NO. 22017 
industry and their firm, but also to "model" different configurations in their minds, to use the business model more as a cognitive tool for modelling alternatives than as a rigid template for constructing their business activities. We suggest that guidelines to help managers to cognitively model their business would provide a strong contribution to stimulating novel, useful and appropriate ways to compete.

In recognizing that much still needs to be done, we ask you as managers, entrepreneurs, consultants, practitioners and academics to contact us with your reactions to this Special Issue. We welcome your ideas and suggestions for how future studies can address the challenges of providing management guidance on reimagining business models and modelling in an increasingly competitive and changing world.

Vincent Mangematin, Aurelio M. Ravarini and Pamela Sharkey Scott

\section{About the Guest Editors}

Professor Vincent Mangematin is Professor and Scientific Director at Grenoble Ecole de Management, France. His research interests lie within innovation and the evolution of technologies, business models and institutions. In analysing the conditions of change and the dynamics of innovation within different industries, Vincent has published in several leading journals including Strategic Management Journal, Research Policy, Journal of International Business Studies and Journal of Business Strategy. Professor Vincent Mangematin can be contacted at: vincent.mangematin@grenoble-em.com

Aurelio M. Ravarini is director of CETIC, Research Center on Information Systems at the LIUC Università Cattaneo (Italy). His research expertise is in strategic information systems, knowledge management systems and information systems development in small- and medium-sized companies. He has published more than 80 papers for international journals, book chapters and conferences proceedings, and serves as Associate Editor for the European Journal of Information Systems. Aurelio M. Ravarini can be contacted at: aravarini@liuc.it

Professor Pamela Sharkey Scott is Professor of International Management at National University of Ireland, Maynooth, Ireland. Her research interests centre on strategy development and modelling in complex organizations. A former senior corporate banker, Pamela is involved in several consulting and policy development roles. She is published in leading international journals including the Journal of International Business Studies, Organization Studies, Technovation and Journal of Business Strategy. Professor Pamela Sharkey Scott can be contacted at: pamela.sharkeyscott@nuim.ie 